\title{
Rate-Adaptive Concurrent Transmission Scheduling Schemes for WPANs with Directional Antennas
}

\author{
Maggie X. Cheng Quanmin Ye Lin Cai
}

\begin{abstract}
We consider the concurrent transmission scheduling problem in a rate-adaptive wireless personal area network. In such networks, the physical layer can adaptively change modulation and coding schemes based on the interference level in the environment and accordingly change the data rate. The scheduling problem is to assign users to time slots so that the total throughput is maximized. The challenge is that the achieved data rate of one flow is limited by the interference from other flows in the same slot, which is unknown until the schedule is known. We propose to discretize data rate into several distinct levels supported by the PHY layer, and then use a linear programming model to find the highest rate level a flow can achieve. The same model is extended to consider a mixture of omni-directional antennas and directional antennas with heterogeneous transmitting power. The simulation results show that the proposed algorithms outperform the previous work for adaptive-rate transmission scheduling in both throughput and fairness.
\end{abstract}

\section{INTRODUCTION}

Wireless personal area networks (WPANs) feature multi-users coexisting in a small area. Due to the short distance, users can communicate with each other directly in a peer-to-peer fashion without involving a relay node. Also due to the short distance, nodes may have harmful interference to each other. However, from a system point of view, having the nodes access the channel one at a time in a serial TDMA manner may be a waste of resource due to the long waiting time each user experiences. The total network throughput may be decreased for not utilizing spectrum spatial reuse. The performance degradation can be significant when it comes to the mm-Wave based WPANs, since at the 60$\mathrm{GHz}$ band oxygen absorption peaks and the transmission range is short, so there is large room for spectrum spatial

Copyright (c) 2014 IEEE. Personal use of this material is permitted. However, permission to use this material for any other purposes must be obtained from the IEEE by sending a request to pubspermissions@ieee.org.

Maggie X. Cheng is with Missouri University of Science and Technology, Computer Science Department; Quanmin Ye is with Nokia Networks; Lin Cai is with the University of Victoria, Electrical and Computer Engineering Department. reuse. To make efficient use of the radio spectrum, a rigorous treatment of the subject is deemed necessary. This motivates the study of finding an optimal schedule for the maximum network throughput.

Although scheduling is a well-studied subject, to optimally schedule transmissions when each user can adjust its data rate according to the signal to interference and noise ratio (SINR) is relatively new. A new dose of challenge is added when the data rate of each user is not known a priori. The achievable data rate is dependent on who else is transmitting, which is not known until the scheduling decision is made.

The rate-adaptive scheduling problem is different from the scheduling problems studied in previous work [1], [2], in which fixed data rates were used for individual flows. The main challenge is to manage the multi-user interference. However, there is no straightforward solution for interference management. How to optimize the scheduling solution for rate-adaptive networks remains an open issue. In this paper, we take an optimization-based approach to determine the concurrent transmission schedule; moreover, we deal with the interference relation in a continuous scale for maximum performance gain, which is superior to the previous work that used a binary conflict relation-based approach [3]. The proposed solution also considers fairness among users. The entire schedule for peer-topeer transmission is updated in each round, which allows real-time applications since the duration of a superframe is long enough to accommodate a schedule update.

The rest of the paper is organized as follows. In Section II, we describe the network model for which the scheduling problem is studied. In Section III, we formally introduce the rate-adaptive scheduling problem. In Section IV, we propose a linear programming model to find the optimal schedule for networks with homogeneous and omni-directional antennas. In Section V, we extend the homogeneous model to networks with heterogeneous transmitting power and different antenna types. We compare the proposed work with previous related work and present simulation results in Section VI. In Section VII, we briefly survey the previous related 
work. Section VIII concludes the paper and points out future research directions.

\section{SYSTEM MODEL}

We consider the model of IEEE 802.15.3 for ultrawideband WPANs. Nodes are deployed in a small region, typically no larger than $10 \mathrm{~m} \times 10 \mathrm{~m}$. Each transmitter can directly transmit to its receiver without using a relay, and this communication pair forms a flow. One of the nodes will be selected as the coordinator of the entire piconet (PNC). Fig. 1 shows the superframe structure. A superframe starts from the Beacon Period (BP), in which the PNC broadcasts network control messages. BP is followed by the Contention Access period (CAP), in which devices send requests to PNC using CSMA/CA. After CAP, it is the Channel Time Allocation Period (CTAP), in which devices communicate with each other in a peer-to-peer fashion using allocated slots.

To accomodata the variable data rate transmission we proposed in this paper, the PNC can use the Beacon Period to announce the schedule together with the rate information for peer-to-peer transmissions, and devices can choose the corresponding coding and modulation schemes based on the schedule and rate information they have received. The PNC receives requests in the current $\mathrm{CAP}$ and announces the schedule at the next BP, thus the PNC can use the entire duration of CTAP to compute the schedule.

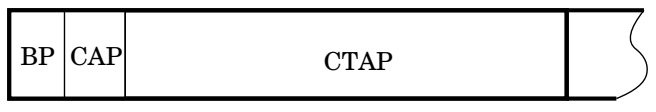

Fig. 1: Superframe structure

Suppose there are $N$ active flows. We name the transmitter of flow $i$ transmitter $i$ and the receiver of flow $i$ receiver $i$. If we assign one flow per slot and arrange transmissions in a round-robin fashion, there will be no multi-user interference. A flow will achieve its highest data rate in the allocated slot, but will have to refrain from access in other slots, so the average data rate of flow $i$ is only $R_{i} / N$, where $R_{i}$ is its achieved data rate in a transmitting slot. If we use CDMA in each slot, and allow multiple flows to transmit in one slot using different codes, it is possible that the total throughput is higher. We assume the codes are pseudorandom sequences, which have a number of good properties including immune to noise and autocorrelation, and low requirement for synchronization.

In this paper, we adopt the same physical layer model used in [3], which uses the $60 \mathrm{GHz}$ mm-Wave unlicensed band, and uses DS-CDMA in each allocated slot. We focus on the scheduling scheme used by the coordinator for slot allocation.

\section{Scheduling Problem for Rate-Adaptive WIRELESS NETWORKS}

Scheduling rate-adaptive flows is fundamentally different from scheduling fixed-rate flows. The latter is easier in that as long as the SINR is above a threshold, it uses a fixed data rate to transmit, while in the former a transmitter varies its data rate according to the received SINR. When the ratio is high, a node increases its data rate to maximize spectrum utilization. If the transmitting power is $P_{T}$, then the received power $P_{R}=\kappa_{1} G_{T} G_{R} P_{T} d^{-\gamma}$, where $\kappa_{1} \propto\left(\frac{\lambda}{4 \pi}\right)^{\gamma}$ is the constant scaling factor corresponding to the reference pathloss, $G_{T}$ and $G_{R}$ are transmit and receive antenna gains respectively, $d$ is the distance between the transmitter and receiver, and $\gamma$ is the path-loss component, usually between 2 and 6 . The noise and interference consists of white Gaussian noise and interference, $I$, from other transmitters. If the white Gaussian noise spectral density is $N_{0}$, then the total noise power is $N_{0} W$. Using the Shannon's theory, the achievable data rate is

$$
R=\kappa_{2} W \log _{2}\left(1+\frac{\kappa_{1} G_{T} G_{R} P_{T} d^{-\gamma}}{N_{0} W+I}\right),
$$

where $\kappa_{2}$ accounts for the efficiency of the transceiver design, and $W$ is the channel bandwidth. When there is only one active flow in the network, the achievable data rate is fully determined by its own transmitting power and constant parameters. However when multiple users share one slot, the achievable data rate for each user is no longer a constant. A transmitter then adapts its data rate based on the current SINR. It is considered rateadaptive because $R$ varies with $I$.

Definition 1 (MTS): The Maximum Throughput Scheduling problem is to find an optimal assignment of flows to slots such that the network throughput is maximized.

For instance, assume that flow 1 and flow 2 have data rates $R_{1}$ and $R_{2}$, respectively, when they each transmit alone. If flow 1 and flow 2 transmit at the same time, the data rate of flow 1 becomes $R_{1}^{2}$ with the superscript indicating the interfering flow. Apparently $R_{1}^{2}<R_{1}$, and $R_{2}^{1}<R_{2}$. The combined throughput is $R_{1}^{2}+R_{2}^{1}$. The optimal solution to the maximum throughput is $\max \left\{R_{1}^{2}+R_{2}^{1}, R_{1}, R_{2}\right\}$. With more flows involved, the optimal solution is selected from a large number of choices exponential to the number of flows. For $N$ flows, the cardinality of the candidate solution set is $\sum_{k=1}^{N}\left(\begin{array}{c}N \\ k\end{array}\right)=2^{N}-1$. Among the $2^{N}-1$ flow assignment options, there exists a flow assignment that provides the maximum total throughput. However, which flows can be put in one slot to maximize the total throughput is a complicated combinatorial optimization problem. We show that the MTS problem is NP-hard 
in the Appendix. Apparently, an exhaustive search algorithm is not practical for large $N$. We henceforth explore a linear programming-based approach.

\section{Linear Programming-Based Transmission SCHEDULING}

The proposed method involves first discretizing data rate to $H$ levels, with each level corresponding to a PHY layer coding and modulation scheme. The lowest data rate, $r_{1}$, is defined as the minimum data rate at which a node is allowed to transmit. In other words, if a node cannot achieve this data rate due to interference, it won't be transmitting. The highest data rate, $r_{H}$, is the maximum data rate a node can achieve when transmitting at the maximum power to a receiver at distance $d=1$ and there is no interference from other flows. The actual data rate of a flow in a transmitting slot is between the two boundaries depending on the interference it receives. Variables and constants used in the linear program are listed as follows.

Variables:

- $0-1$ integer variable $u_{i}=1$ if flow $i$ uses the current slot to transmit; $=0$ otherwise.

- 0-1 integer variable $t_{i, h}=1$ if flow $i$ uses the current slot to transmit at rate level $h ;=0$ otherwise.

- Real-valued variable $R_{i}$ is the achieved data rate of flow $i$ in the current slot. If flow $i$ is not transmitting, $R_{i}=0$.

Constants:

- $N$ is the number of flows.

- $K$ is the number of slots.

- $W$ is spectrum bandwidth in $\mathrm{MHz}$.

- $\gamma$ is the path-loss exponent.

- $\kappa_{1}$ is a constant dependent on wavelength, $\kappa_{1} \propto$ $\left(\frac{\lambda}{4 \pi}\right)^{\gamma}$.

- $\kappa_{2}$ is the coefficient describing the efficiency of the transceiver design.

- $r_{h}$ for $h=1 . . H$ are the discretized data rates. $r_{1}$ is the the minimum rate, and $r_{H}$ is the maximum rate. $H$ is the number of levels.

- $S I N R_{h}$ is the signal to interference and noise ratio threshold to achieve data rate $r_{h}$. Using equation $r_{h}=\kappa_{2} W \log _{2}\left(1+S I N R_{h}\right), S I N R_{h}$ can be calculated as follows: $S I N R_{h}=2^{r_{h} /\left(\kappa_{2} W\right)}-1$ for a given data rate $r_{h}$.

- $G_{T}(i)$ is the omni-directional antenna gain of transmitter $i, G_{R}(i)$ is the omni-directional antenna gain of receiver $i$. For homogeneous model, $G_{T}(i)=$ $G_{R}(i)=\eta \times 1$, where $\eta$ is the antenna radiation efficiency.

- $b$ is cross-correlation between two concurrent transmissions in a CDMA context, also called multiuser interference (MUI) factor.

- $N_{\infty}$ is a large positive number.

\section{A. A High Throughput Scheduling Algorithm (LP)}

Let $k$ be the current slot number. $R_{i}^{(k)}$ is the sum of data rates from slots 1 to $k$, for $k=1, \ldots, K$. Upon termination, we get $R_{i}^{(K)} / K$, the average data rate of flow $i$ over $K$ slots. Initially we set $k=1$, and $R_{i}^{(0)}=$ $0, \forall i$

Algorithm $L P$

- Step 1: Pick the flow that has the lowest throughput:

$$
i^{*}=\arg \min _{i} R_{i}^{(k-1)}
$$

then set $u_{i^{*}}=1$. For the first iteration, since all flows have $R_{i}^{(0)}=0$, pick a flow randomly.

- Step 2: Solve the following linear program, and obtain data rate $R_{i}$ and slot assignment $u_{i}$ at the current slot:

Maximize

$$
\sum_{i=1}^{N} R_{i}
$$

Subject to

$$
\begin{aligned}
& u_{i^{*}}=1 \\
& u_{i} \leq \sum_{h=1}^{H} t_{i, h}, \quad \forall i \\
& u_{i} \geq t_{i, h}, \quad \forall i, \forall h \\
& \sum_{h=1}^{H} t_{i, h} \leq 1, \quad \forall i \\
& R_{i} \leq \sum_{h=1}^{H} t_{i, h} r_{h}, \quad \forall i \\
& N_{\infty}\left(1-t_{i, h}\right)+\left(\kappa_{1} G_{i, i} P_{T} d_{i, i}^{-\gamma} / S I N R_{h}\right) \geq \\
& N_{0} W+b \sum_{l \neq i}\left(u_{l} \kappa_{1} G_{T}(l) G_{R}(i) P_{T} d_{l, i}^{-\gamma}\right), \quad \forall i, \forall h
\end{aligned}
$$

- Step 3: update $R_{i}^{(k)}$ :

$$
R_{i}^{(k)}=R_{i}^{(k-1)}+R_{i}, \quad \forall i
$$

- Step 4: $k=k+1$. While $k \leq K$, repeat steps 1-3.

- Return the average data rate of each flow $R_{i}^{(K)} / K$, and network throughput $\sum_{i=1}^{N} R_{i}^{(K)} / K$.

Inequality (2b) requires that if $t_{i, h}=0$ for all $h$, then $u_{i}=0$, which means if flow $i$ is not using any valid data rate to transmit, then flow $i$ is not transmitting in slot $k$ at all. Inequality (2c) requires that if $t_{i, h}=1$ for some $h$, then $u_{i}=1$, which states that if flow $i$ is using some 
data rate $r_{h}$ to transmit, then flow $i$ is transmitting in slot $k$. Inequality $(2 \mathrm{~d})$ requires that a flow either use one rate level to transmit or not transmit at all. Inequality (2e) states that if flow $i$ is using level $h$ then the achieved data rate is $r_{h}$ in this slot. (2f) requires that if flow $i$ has data rate $r_{h}$ in slot $k$, then the signal to interference and noise ratio must be at least $S I N R_{h}$; otherwise if $t_{i, h}=0$, the inequality is automatically satisfied for having a large positive constant at the left hand side.

Formulating the MTS problem into a Mixed Integer Program (MIP) does not make the original problem easier to solve, since it is still an NP-hard problem, but it does provide a way to approach the optimal solution. The MIP can be solved by first relaxing it to a realvalued linear program and then rounding fractions to integers. The heuristics yields a feasible schedule, from which the actual data rates are calculated according to the Shannon's theory. Most solvers (i.e., lp_solve) have built-in ability to do LP-relaxation and rounding. It takes only $O\left(n^{3}\right)$-time to solve a real-valued linear program with $n$ variables, much lower than the exponential-time exhaustive search algorithm.

\section{B. Throughput-Fairness Tradeoff Design (LP-Fair)}

Although the algorithm $L P$ does have some control over fairness by allowing the flow with the lowest data rate to transmit in the next slot, the achieved data rate in the next slot still depends on the interference from other transmitters. To improve fairness, we introduce a coefficient $c_{i}\left(c_{i}>0\right)$ for each flow $i$ and use the following objective function in Step 2. We call this algorithm LP-Fair.

\section{Maximize}

$$
\sum_{i=1}^{N} c_{i} R_{i}
$$

In the first slot $(k \stackrel{i=1}{=})$, we set $c_{i}=1, \forall i$. When $k \geq 2, c_{i}$ reflects the priority to transmit for each node. To decide the coefficient $c_{i}$, we need a function $f\left(R_{1}^{(k-1)}, \ldots, R_{N}^{(k-1)}\right): \mathcal{R}^{N} \rightarrow \mathcal{R}^{N}$ that has the following property: if $R_{i}^{(k-1)}<R_{j}^{(k-1)}$, then $c_{i}>c_{j}$, so that in the next round, node $i$ has better chance to transmit than node $j$. There are infinite number of functions that have this property. For instance,

1) $c_{i}=\operatorname{rank}\left(R_{i}^{(k-1)}\right)$. The largest rate has rank 1 , and the smallest rate has rank $N$.

2) $c_{i}=\sum_{i=1}^{N} R_{i}^{(k-1)} /\left(R_{i}^{(k-1)}+1\right)$

We choose to use the first one in this paper for its simplicity, although others can do the job equally well. To see how $c_{i}$ regulates fairness, we consider the 5flow network in Fig. 2. In the first slot, flows 1, 2 and 5 transmitted, and flows 3 and 4 did not, so we assign $c=$ $\{1,1,3,3,2\}$ for flows 1 to 5 (same number indicates there is a tie). In the second round, flow 4 was selected as the seed $\left(i^{*}=4\right)$, and flows 4 and 5 transmitted, then we set $c=\{3,3,4,2,1\} . L P$ already has fairness consideration by choosing the seed of the next round. The use of coefficient $c_{i}$ can further improve fairness over $L P$ by assigning different priorities. The effect of using $c_{i}$ is observed at slot 2, when both $L P$ and $L P$ Fair selected flow 4 as the seed, $L P$ would choose flow 2 to transmit together with flow 4, LP-Fair would choose flow 5 to transmit together with flow 4 , because $c_{5}=$ 2 , and $c_{2}=1$, therefore flow 5 has higher priority to transmit than flow 2 .

\section{A Benchmark for Maximum Throughput}

To assess the effectiveness of the proposed algorithm, we present a benchmark model. The benchmark model is a global optimization model that tries to schedule transmissions for all slots in one linear program, with an objective of maximizing the total throughput. All variables need to add one more dimension $k$ to indicate slot number. The following notations are used for variables:

- $u_{k, i}=1$ if flow $i$ uses slot $k$ to transmit; $=0$ otherwise.

- $t_{k, i, h}=1$ if flow $i$ uses slot $k$ to transmit at rate level $h ;=0$ otherwise.

- $R_{k, i}$ is the achieved data rate of flow $i$ in slot $k$. If node $i$ is not transmitting in slot $k, R_{k, i}=0$.

\section{Maximize}

$$
\frac{1}{K} \sum_{i=1}^{N} \sum_{k=1}^{K} R_{k, i}
$$

\section{Subject to}

$$
\begin{aligned}
& u_{k, i} \leq \sum_{h=1}^{H} t_{k, i, h}, \quad \forall k, \forall i \\
& u_{k, i} \geq t_{k, i, h}, \quad \forall k, \forall i, \forall h \\
& \sum_{h} t_{k, i, h} \leq 1, \quad \forall k, \forall i \\
& R_{k, i} \leq \sum_{h=1}^{H} t_{k, i, h} r_{h}, \quad \forall k, \forall i \\
& N_{\infty}\left(1-t_{k, i, h}\right)+\left(\kappa_{1} G_{i, i} P_{T} d_{i, i}^{-\gamma} / S I N R_{h}\right) \geq \\
& N_{0} W+b \sum_{l \neq i}\left(u_{k, l} \kappa_{1} G_{T}(l) G_{R}(i) P_{T} d_{l, i}^{-\gamma}\right)
\end{aligned}
$$

Solving this optimization problem will lead to the slot assignment for all slots. We call this algorithm Aggregate.

In addition to pursuing the maximum throughput, sometimes there is a strict requirement for fairness among flows. We adopt a widely used method to address 
fairness requirement, and call the algorithm with fairness control Aggregate-Fair.

- Each individual flow must achieve at least $p$ fraction of the total throughput, where $0 \leq p \leq$ $1 / N$. We add the following constraint:

$$
\sum_{k} R_{k, i} \geq p \sum_{i} \sum_{k} R_{k, i}, \quad, \forall i
$$

The solution to the linear programming model defined by (4)-(5e) leads to the maximum throughput, but it cannot be efficiently solved for large networks. With the additional fairness constraint, it is more complex. In the next section, we use it as a benchmark to evaluate the performance of the slot-by-slot methods $L P$ and LP-Fair.

\section{Comparison to the Benchmark}

We show a network with ten nodes and five flows deployed on a $10 \mathrm{~m} \times 10 \mathrm{~m}$ square region. Transmission power $P_{T}$ is set to $10 \mathrm{~mW}$. The number of levels for data rate is five $(\mathrm{H}=5)$. Transmitters and receivers are shown in Fig. 2. Aggregate-Fair algorithm is used with $p=1 / 10$. Transmission schedule is shown in TABLE I. The Aggregate model would schedule flow 2 and flow 3 in every slot, and TDMA would schedule one flow per slot, but the slot-by-slot model $L P$ would schedule flow 1 , flow 2, and flow 3 in the first slot, then flow 2 and flow 4 in the second slot, and so on. Throughput result is shown in TABLE II. Comparing $L P$ with Aggregate, $L P$ has $3.1 \%$ throughput loss; Comparing LP-Fair with Aggregate-Fair, LP-Fair has only 1.1\% throughput loss since the fairness constraint also reduces total throughput for Aggregate-Fair.

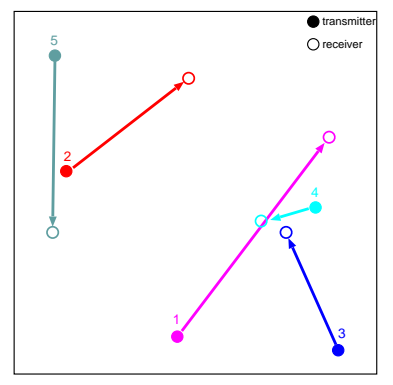

Fig. 2: A network of 10 nodes, 5 flows.

Running-time Comparison: Compared to the benchmark models, which schedule all slots in one linear program, the proposed slot-by-slot models reduce the number of variables by a factor of $K$ ( $K$ is the number of slots). Solving the real-valued linear program is in the order of $O\left(n^{3}\right)$ where $n$ is the number of variables. The slot-by-slot models solve the smaller linear program for $K$ times, so the overall running time is still reduced by a factor of $K^{2}$ from the benchmark model. The running-time efficiency is achieved with a slight tradeoff in throughput performance.

\begin{tabular}{|c||c|c|c|c|c|}
\hline Slot & Agg & Agg-Fair & LP & LP-Fair & TDMA \\
\hline \hline $\mathbf{1}$ & 2,3 & $1,2,3$ & $1,2,3$ & $1,2,5$ & 1 \\
\hline $\mathbf{2}$ & 2,3 & 4,5 & 2,4 & 4,5 & 2 \\
\hline $\mathbf{3}$ & 2,3 & $1,2,3$ & $2,3,5$ & $2,3,5$ & 3 \\
\hline $\mathbf{4}$ & 2,3 & 4,5 & $1,2,3$ & $1,2,3$ & 4 \\
\hline $\mathbf{5}$ & 2,3 & $1,2,3$ & 2,4 & 2,4 & 5 \\
\hline
\end{tabular}

TABLE I: Transmission scheduling

\begin{tabular}{|c||c|c|c|c|c|}
\hline Flow & Agg & Agg-Fair & LP & LP-Fair & TDMA \\
\hline \hline $\mathbf{1}$ & 0 & 1023.52 & 682.34 & 911.02 & 341.21 \\
\hline $\mathbf{2}$ & 3706.23 & 1690.68 & 2705.09 & 2147.81 & 563.62 \\
\hline $\mathbf{3}$ & 3706.23 & 1519.69 & 1646.33 & 1139.76 & 633.27 \\
\hline $\mathbf{4}$ & 0 & 1663.33 & 2049.97 & 1856.65 & 1025.09 \\
\hline $\mathbf{5}$ & 0 & 1241.39 & 99.41 & 1005.03 & 497.07 \\
\hline \hline$\sum$ & 7412.46 & 7138.68 & 7183.14 & 7060.27 & 3060.26 \\
\hline
\end{tabular}

TABLE II: Flow rate and network throughput (Mbps)

\section{E. Performance Gap}

The proposed linear programs all have integer variables in them, and the exact solutions for integer or mixed integer programs are NP-hard to compute. To understand how close the results are from the optimal solutions, we need to know the optimal solutions. However, the dilemma is that we do not have an efficient method to compute the optimal solutions. Since these problems are all maximization problem, a reasonable approach is to compare the obtained solutions with the upper bounds of the optimal solutions. Taking program (4)-(5e) as an example, we can find the upper bound of the optimal solution by computing the following realvalued linear program:

- real-valued variable $u_{k, i}, 0 \leq u_{k, i} \leq 1$

- real-valued variable $t_{k, i, h}, 0 \leq t_{k, i, h} \leq 1$

- Real-valued variable $R_{k, i}$ is the achieved data rate of flow $i$ in slot $k$. If node $i$ is not transmitting in slot $k, R_{k, i}=0$.

\section{Maximize}

$$
\frac{1}{K} \sum_{i=1}^{N} \sum_{k=1}^{K} R_{k, i}
$$

\section{Subject to}

$$
\begin{aligned}
& u_{k, i} \leq \sum_{h=1}^{H} t_{k, i, h}, \quad \forall k, \forall i \\
& u_{k, i} \geq t_{k, i, h}, \quad \forall k, \forall i, \forall h \\
& \sum_{h} t_{k, i, h} \leq 1, \quad \forall k, \forall i \\
& R_{k, i} \leq \sum_{h=1}^{H} t_{k, i, h} r_{h}, \quad \forall k, \forall i \\
& N_{k, i}=N_{0} W+b \sum_{l \neq i}\left(\kappa_{1} G_{T}(l) G_{R}(i) P_{T} d_{l, i}^{-\gamma}\right), \quad \forall k, \forall i
\end{aligned}
$$




$$
\begin{aligned}
& N_{k, i}\left(1-t_{k, i, h}\right)+\left(\kappa_{1} G_{i, i} P_{T} d_{i, i}^{-\gamma} / S I N R_{h}\right) \geq \\
& N_{0} W+b \sum_{l \neq i}\left(u_{k, l} \kappa_{1} G_{T}(l) G_{R}(i) P_{T} d_{l, i}^{-\gamma}\right), \quad \forall k, \forall i, \forall h
\end{aligned}
$$

Program (6)-(7f) is solvable in polynomial time. With $n$ variables, the running time complexity is $O\left(n^{3}\right)$. If the objective value of the optimal solution to (6)-(7f) is $O P T_{\text {rel }}$, and the objective value of the optimal solution to (4)-(5e) is $O P T_{i n t}$, then

$$
O P T_{\text {rel }} \geq O P T_{\text {int }}
$$

since the optimal solution of (4)-(5e) is a feasible solution of (6)-(7f), and $O P T_{\text {rel }}$, by definition, is the largest value among all feasible solutions.

If the objective solution from the proposed heuristics is $S O L_{A}$, which is obtained by solving the real-valued program and then rounding up fractional values to integer values, then we have

$$
S O L_{A} \leq O P T_{\text {int }} \leq O P T_{\text {rel }}
$$

The gap between $S O L_{A}$ and $O P T_{i n t}$ is certainly smaller than the gap between $S O L_{A}$ and $O P T_{\text {rel }}$. So we have

$$
\frac{O P T_{\text {int }}}{S O L_{A}} \leq \frac{O P T_{\text {rel }}}{S O L_{A}}
$$

The LHS is the performance ratio that we want to know, but is not available; the RHS is the upper bound of the performance ratio. The experiment results show that the upper bound of the performance ratio is around $1.5 \sim$ 1.6 for the following test cases:

\begin{tabular}{|c||c|c|c|c|}
\hline Flow Number & 5 flows & 10 flows & 15 flows & 20 flows \\
\hline Ratio $\frac{O P T_{r e l}}{S O L_{A}}$ & 1.53 & 1.542 & 1.55 & 1.57 \\
\hline
\end{tabular}

TABLE III: The upper bound of the performance ratio.

The actual performance ratio is smaller than the data shown in the table. We conclude that the performance of the proposed heuristics is close to the optimal solution.

\section{Extension to Directional Antenna And Heterogeneous Transmitting Power}

In this section, we consider networks with directional antennas and non-uniform transmission power. There are several benefits of using directional antenna: it increases the signal strength at the intended receiver while at the same time reduces interference to others. Although WPANs usually have small network coverage area and there is no need to increase transmission range, we can achieve much higher network throughput by using directional antenna. The new interference relation will not only depend on node positions but also the beamwidth and orientation of the axes of the radiation sectors. A directional antenna pattern consists of a main lobe with gain $G_{M}=\eta \frac{2 \pi}{\theta}$ and beamwidth $\theta$ and side lobes with gain $G_{S}=(1-\eta) \frac{2 \pi}{2 \pi-\theta}$ and aggregated beamwidth $2 \pi-\theta$. Let $G_{T}(i, j)$ be the transmit antenna gain between transmitter $i$ and receiver $j ; G_{R}(i, j)$ is the receiver antenna gain between transmitter $i$ and receiver $j$. If transmitter $i$ is omni-directional, then $G_{T}(i, j)$ is the same as $G_{T}(i)$ in Section IV regardless of the location of receiver $j$. Similarly, if receiver $j$ is omnidirectional, then $G_{R}(i, j)=G_{R}(j)$ regardless of the location of transmitter $i$. When transmitter (or receiver) $i$ has a directional antenna, in order to achieve the maximum directivity gain, we can make receiver (or transmitter) $i$ align with transmitter (or receiver) $i$ 's main lobe axis.

In heterogeneous networks, in addition to using different types of antennas, nodes can also have different transmit power. Let $P_{T}(i)$ be the transmit power of flow $i$, then the received power of flow $i$ is calculated as

$$
P_{R}(i)=\kappa_{1} G_{T}(i, i) G_{R}(i, i) P_{T}(i) d_{i, i}^{-\gamma}
$$

The interference power from transmitter $j$ to receiver $i$ is

$$
I_{j, i}=\kappa_{1} b G_{T}(j, i) G_{R}(j, i) P_{T}(j) d_{j, i}^{-\gamma}
$$

With the above substantiation, we can replace inequality (2f) with the following in the linear program for $L P$ and LP-Fair.

$$
\begin{gathered}
N_{\infty}\left(1-t_{i, h}\right)+\left(\kappa_{1} G_{T}(i, i) G_{R}(i, i) P_{T}(i) d_{i, i}^{-\gamma} / S I N R_{h}\right) \geq \\
N_{0} W+b \sum_{l \neq i}\left(u_{l} \kappa_{1} G_{T}(l, i) G_{R}(l, i) P_{T}(l) d_{l, i}^{-\gamma}\right), \quad \forall i, \forall h .
\end{gathered}
$$

Case 1: Omni-directional Transmitter and Receiver

If flow $i$ has an omni-directional transmitter and an omni-directional receiver, then $G_{T}(i, i)=G_{T}(i)$ and $G_{R}(i, i)=G_{R}(i)$. For other interfering transmitters $l \neq i$,

$G_{T}(l, i)= \begin{cases}G_{T}(l), & \text { if } \operatorname{Tx} l \text { is omni-directional; } \\ G_{M}, & \text { if } \operatorname{Tx} l \text { is directional and } \operatorname{Rx} i \text { is } \\ & \text { inside the main lobe of } \operatorname{Tx} l ; \\ G_{S}, & \text { if } \operatorname{Tx} l \text { is directional and } \operatorname{Rx} i \text { is } \\ & \text { not inside the main lobe of } \operatorname{Tx} l .\end{cases}$

Since receiver $i$ is omni-directioanl, $G_{R}(l, i)=$ $G_{R}(i), \forall l$.

\section{Case 2: Directional Transmitter and Omni-directional} Receiver

Transmitter $i$ is directional with $G_{T}(i, i)=G_{M}$. Receiver $i$ is omni-directional with $G_{R}(i, i)=G_{R}(i)$. For other interfering transmitters $l \neq i, G_{R}(l, i)=G_{R}(i)$, and we follow the same discussion from Case 1 for $G_{T}(l, i)$. 
Case 3: Omni-directional Transmitter and Directional Receiver

Transmitter $i$ is omni-directional with $G_{T}(i, i)=G_{T}(i)$. Receiver $i$ is directional with $G_{R}(i, i)=G_{M}$. For other interfering transmitters $l \neq i, G_{T}(l, i)$ follows Case 1 . Since receiver $i$ is directional, $G_{R}(l, i)$ will depend on whether transmitter $l$ is located within the main lobe of the receiver $i$.

$G_{R}(l, i)= \begin{cases}G_{M}, & \text { if } l \text { is inside the main lobe of } i \\ G_{S}, & \text { otherwise. }\end{cases}$

\section{Case 4: Directional Transmitter and Receiver}

Transmitter $i$ is directional with $G_{T}(i, i)=G_{M}$. Receiver $i$ is directional with $G_{R}(i, i)=G_{M}$. For other transmitters $l \neq i, G_{T}(l, i)$ follows Case 1 , and $G_{R}(l, i)$ follows Case 3.

\section{Vi. Performance Evaluation}

We evaluate the proposed schemes on the number of concurrent transmissions, network throughput, and fairness through simulation. The algorithms using objective functions (1) and (3) are named $L P$ and LP-Fair, respectively. The proposed schemes are compared with the REX scheme in [3] and the serial TDMA scheme using the same network setup.

The networks are set up on a $10 \times 10 \mathrm{~m}^{2}$ area. A total of $2 N$ nodes are randomly deployed in the square region, from which $N$ nodes are randomly selected as transmitters and the remainders are designated as receivers to form $N$ active flows. We have tested networks of different sizes with $N=5 \sim 80$.

In all simulations we have used the following settings:

- The number of slots $K$ in a frame is the same as the number of flows $N$. This is not a requirement by the proposed algorithms, but a requirement of the serial TDMA, which assigns one flow per slot in a TDMA frame.

- For transmission power $P_{T}$, with omni-to-omni transmissions, we vary the transmission power from 0.4 to $165 \mathrm{~mW}$, which is equivalent to having exclusive region radius $r_{0}=0.5 \mathrm{~m}$ to $10 \mathrm{~m}$ in $R E X$ [3]; if directional antennas are involved, we use a constant transmission power $P_{T}=10 \mathrm{~mW}$ and vary the beamwidth $\theta$ from $6^{\circ}$ to $90^{\circ}$ with increment $\Delta \theta=6^{\circ}$.

- Data rate levels $H$ is set to 5 .

- Other parameters: $\gamma=4, W=500 \mathrm{MHz}, \eta=0.9$, $b=10^{-2}, N_{0}=-114 d B W / M H z, \kappa_{1}=-51 d B$, $\kappa_{2}=1, N_{\infty}=10^{5}$.

\section{A. On Concurrent Transmissions}

For a serial TDMA, the number of concurrent transmissions is exactly one. For REX, LP and LP-Fair, the number of concurrent transmissions in each slot is in general larger than one and non-uniform, so the average over all $K$ slots is used. If the number of transmissions in slot $k$ is $x_{k}$, then $X=\frac{1}{K} \sum_{k=1}^{K} x_{k}$ is used in the plots. For each point in the plot, we run 10 randomly generated test cases and calculate the average.

In Fig. 3, we show the average number of concurrent transmissions by $L P$ and $R E X$ with 10,30 , and 40 flows. In Fig. 3(a) the number of concurrent transmissions decreases with transmission power due to the cross-flow interference, and increases with the number of flows due to having more choices to select from. In Fig. 3(b) and (c) when directional antennas are used, we observed that concurrency decreases with beamwidth since larger beamwidth causes larger cross-flow interference.

\section{B. On Throughput}

Fig. 4 shows network throughput achieved on the same network instances used in Fig. 3. In Fig. 4(a), as we increase the transmission power, there is an initial climbing phase from $0.4 \mathrm{~mW}$ to around $3.75 \mathrm{~mW}$; after this point, network throughput decreases with transmission power due to stronger cross-flow interference. In Fig. 4(b) and (c), we use constant transmission power 10 $\mathrm{mW}$, and network throughput decreases with beamwidth due to larger interference area.

We further investigated the impact of having more flows on network performance by using a fixed transmission power $10 \mathrm{~mW}$ and a fixed beamwidth $30^{\circ}$. The results in Fig. 5 confirmed the increasing trend of throughput with the number of flows, and the conclusion is true for both omnidirectional and directional antennas.

We also evaluated the algorithms in heterogeneous networks by randomly selecting either an omnidirectional or a directional antenna for each node while keeping the fraction of nodes with directional antennas a fixed constant $p$. We ensure that if a transmitter is directional, it must face the receiver; if a receiver is directional, it must face the transmitter. We compare network throughput resulting from $L P$ and REX. Fig. 6(a) is the result with beamwidth $\theta=30^{\circ}$, and (b) with $\theta=45^{\circ}$. The result shows $L P$ outperforms REX in every single case and the throughput gain is increasing with number of flows and the percentage of directional antennas. With $\theta=30^{\circ}$, at $p=20 \%$, the throughput gain of $L P$ over $R E X$ is from $4 \%$ to $45 \%$, increasing with the number of flows; and at $p=80 \%$, the throughput gain is from $9 \%$ to $65 \%$. With $\theta=45^{\circ}$, the throughput gain is relatively smaller and the range of throughput gain is 


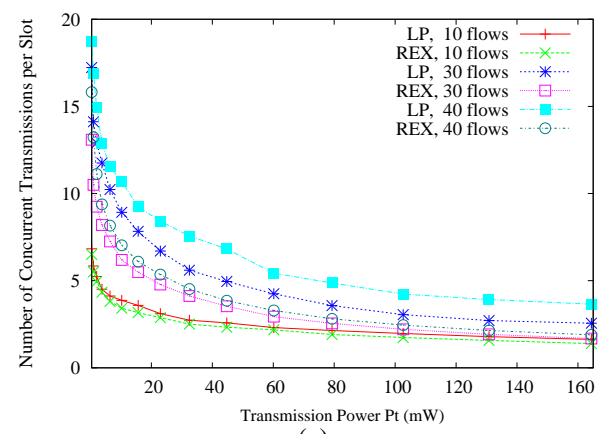

(a)

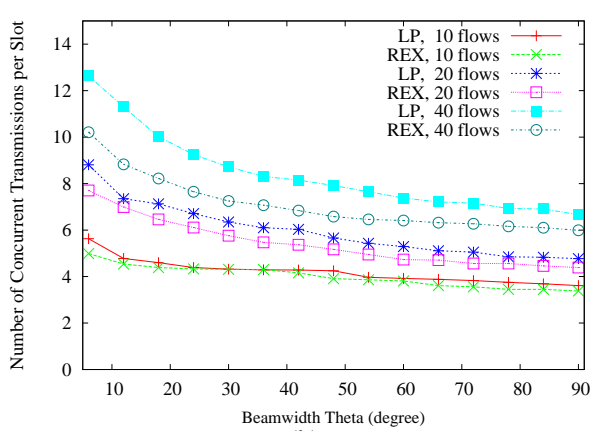

(b)

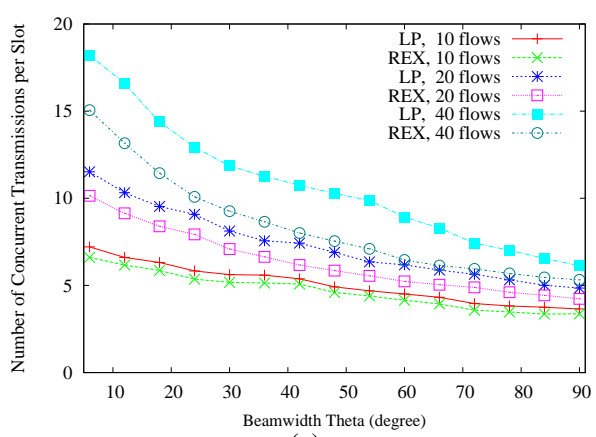

(c)

Fig. 3: Concurrent transmissions, (a) Omni-Omni, (b) Omni-Directional, (c) Directional-Directional.

from $4 \%$ to $28 \%$ at $p=20 \%$ and from $8.5 \%$ to $64 \%$ at $p=80 \%$.

\section{On Fairness}

To evaluate peer fairness, we use Jain's fairness index. Let $y_{i}$ be the measurement for flow $i$, then Jain's fairness is computed as $\left(\sum_{i=1}^{N} y_{i}\right)^{2} /\left(N \sum_{i=1}^{N} y_{i}^{2}\right)$. We first compare resource allocation fairness by using the number of slots allocated to each flow as measurement, then compare data rate fairness by using the per flow data rate as measurement. We call the two indices slot-index and rate-index respectively. Apparently the serial TDMA scheme has a perfect slot-index of 1 .

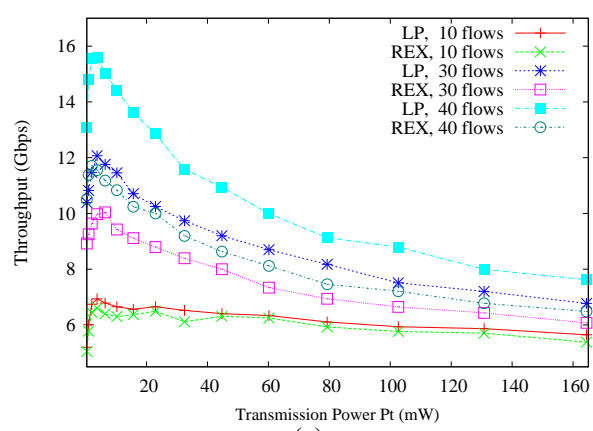

(a)

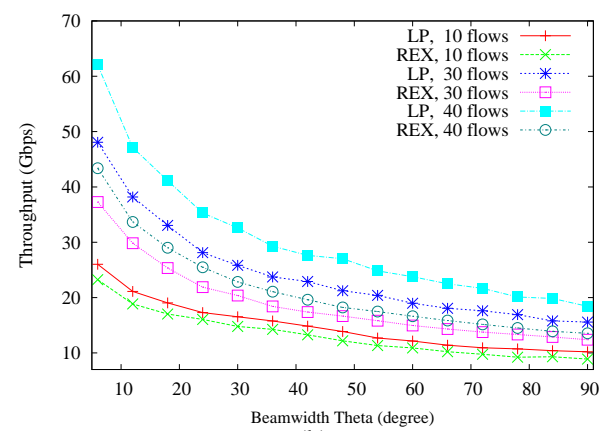

(b)

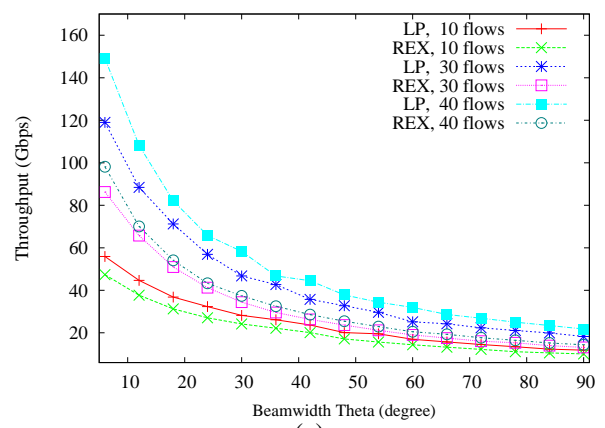

(c)

Fig. 4: Throughput, (a) Omni-Omni, (b) OmniDirectional, (c) Directional-Directional.

We compare $L P, L P$-Fair and REX on their slot-index and rate-index. Since there exists a tradeoff between network throughput and peer fairness, we also included network throughput to show the overall performance of these schemes. We use fixed transmission power $P_{t}=10$ for all cases, and use $\theta=30^{\circ}$ for directional antennas. Fig. 7 shows the cases in which transmitter and receiver both use omni-directional antennas, and Fig. 8 shows the cases in which transmitter and receiver both use directional antennas. It is shown that LP-Fair has the best fairness performance and the second best throughput performance; $L P$ has the best throughput performance, and the second best fairness performance. Both $L P$ and $L P$-Fair outperform $R E X$ in throughput and 


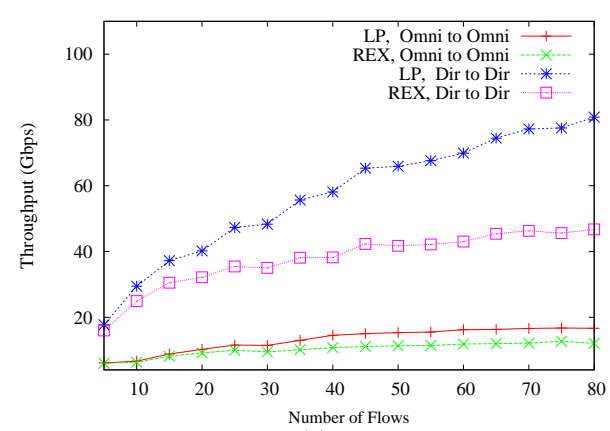

(a)

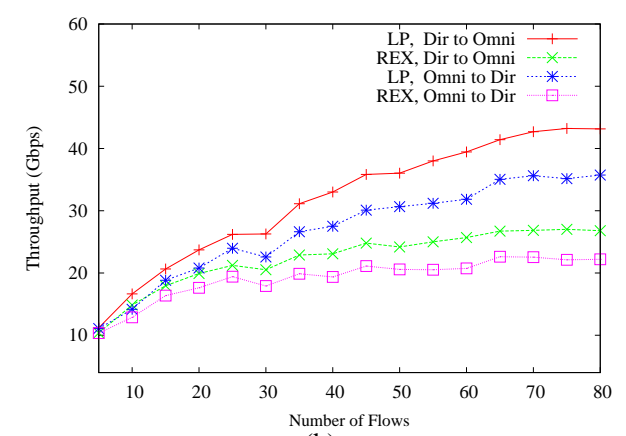

(b)

Fig. 5: Throughput, (a) Tx and Rx use the same type of antenna, (b) Tx and Rx use different types of antennas.
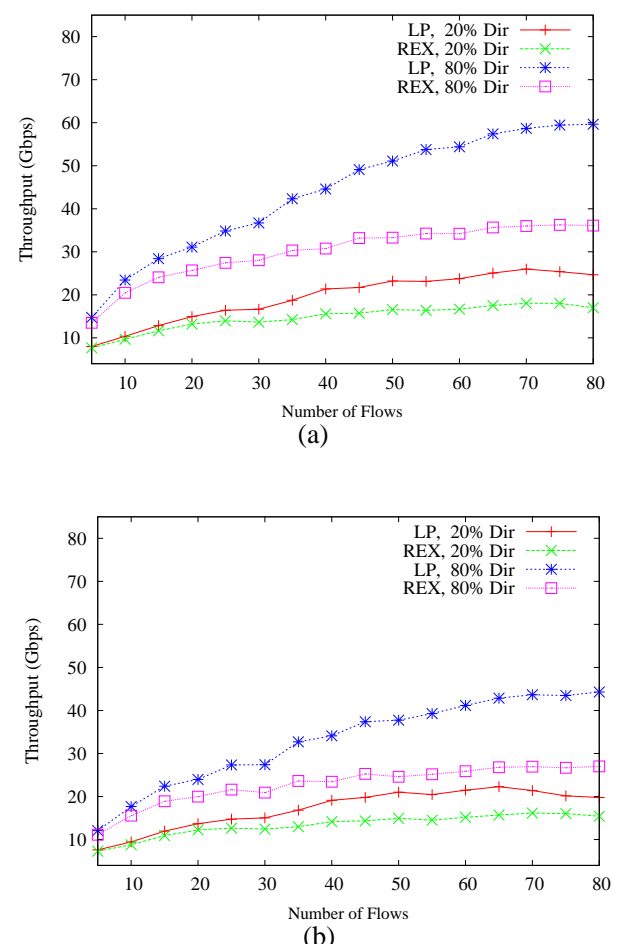

Fig. 6: Throughput result for heterogeneous networks with $20 \%$ or $80 \%$ directional antennas, (a) $\theta=30^{\circ}$, (b) $\theta=45^{\circ}$.

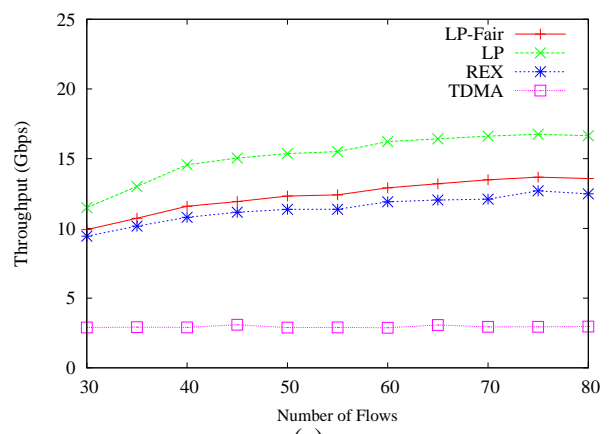

(a)

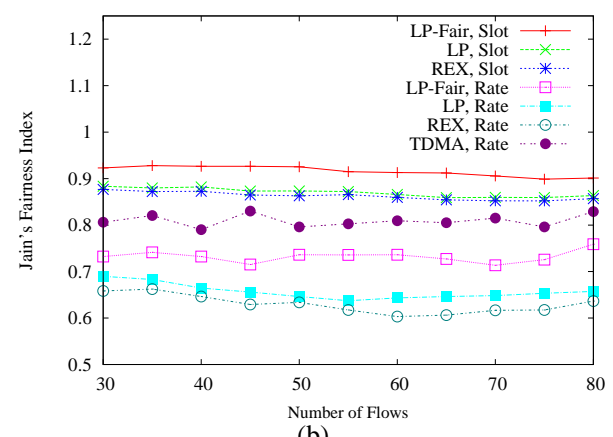

Fig. 7: Fairness result for Omni-Omni transmissions, (a) Network throughput, (b) Jain's index

fairness simultaneously.

It is further observed that every scheme has a larger slot-index than its rate-index. Since slot allocation is our means, slot-index can be easily controlled; but data rate is the outcome of using slot allocation, so rate-index can only be indirectly controlled. LP-Fair outperforms the other two schemes in both slot-index and rate-index, and it is noteworthy to point out that LP-Fair has the smallest gap between slot-index and rate-index, which indicates effective control over peer fairness.

\section{RELATED WORK}

Scheduling is a major research problem in wireless communication and has received extensive study since the earliest days of wireless communication. Different network models have been considered. Some deal with peer-to-peer communication in personal area networks [3]-[6], some deal with one-hop communication in cellular networks [7], and some deal with multi-hop communication in ad hoc networks [1], [2], [8]. The performance consideration is mainly throughput and fairness among users.

In [9]-[11], a conflict graph is constructed to bound the mutual interference so the SINR of the tagged transmission can be above the threshold for a predetermined data rate. As a result, fixed data rates for all transmitter- 


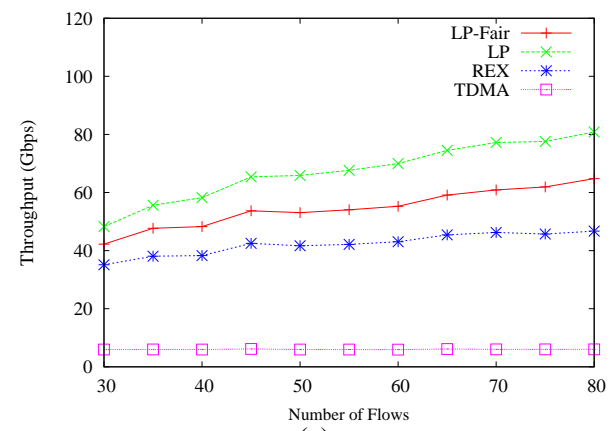

(a)

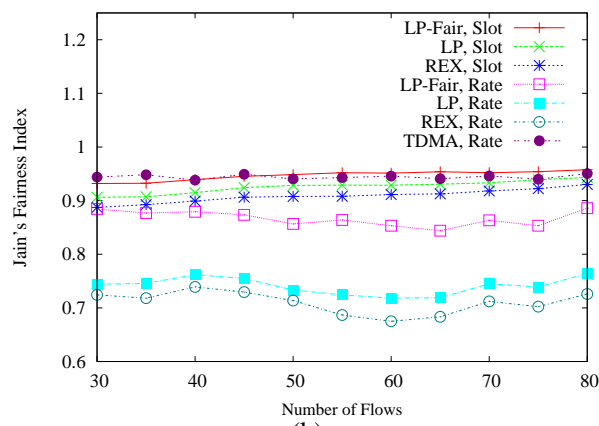

(b)

Fig. 8: Fairness result for Dir-Dir transmissions, (a) Network throughput, (b) Jain's index

receiver pairs are used throughout the communication session. [12] is another example in which SINR is considered during scheduling but only used as a threshold for a fixed data rate. These works are fundamentally different from ours which treats data rate as a variable. In broadband wireless systems such as UWB and mmWave wireless networks, the transmitter can adjust the data rate according to the SINR. With such rate-adaptive property, these conflict graph based solutions are no longer optimal. To schedule concurrent transmissions in a rate-adaptive network, [13]-[15] proposed scheduling solutions based on the exclusive region concept, which reserves an area for each flow to avoid harmful mutual interference. It is found that these concurrent scheduling can result in much higher network throughput than TDMA. [4] further proposed a global search-based algorithm to achieve higher throughput with concurrent transmissions.

Proportionally fair scheduling [16], [17] has been studied for simplified network models in [18]-[21], in which at most two users are allocated to any slot. The cardinality of the candidate solution set is polynomial, and therefore it renders a polynomial-time algorithm. Different from previous work, this paper deals with unbounded users in any slot. The optimal solution is selected from an exponential-sized candidate set, and it is an NP-hard problem.

The most related work to this paper is [3] in which the same network model is considered, where nodes can use directional antennas and data rate can be adapted to the received SINR. The method used in [3] is to derive a sufficient condition that ensures the aggregated data rate from concurrent transmissions be higher than the would-be average data rate in a serial TDMA scheme, and then compute an exclusive region for each flow based on this sufficient condition and antenna directivity. The scheduling process starts from randomly selecting a flow, and then adds flows one-by-one if the new flow and existing flows are out of each other's exclusive region. The benefit of this approach is its simplicity, since it is easy to determine if a node is inside a region; the drawback is that it has turned a continuous-scaled interference relation into a binary relation - transmitters inside the region are forbidden to transmit, and those outside of the region are allowed to transmit. In fact, multiple transmitters outside of the exclusive region have accumulated effect on the receiver and their aggregated interference maybe even higher than from the one inside the exclusive region, but they could be allowed to transmit at the same time if their exclusive regions are mutually exclusive. These are the reasons for performance loss. [3] is an improvement and extension of earlier works that use the concept of exclusive region [13]-[15].

\section{CONCLUSION}

We considered scheduling concurrent transmissions in a variable data rate WPAN. Linear programmingbased algorithms for maximum throughput with fairness consideration have been proposed. The simulation results showed significant improvement over TDMA and earlier work with concurrent transmissions. The performance gain increases with the directivity of the antenna and the percentage of nodes with directional antennas in the network. The proposed algorithms $L P$ Fair is the best in fairness and the second best in throughput, and $L P$ is the best in throughput and the second best in fairness; the two algorithms outperform previous work REX in both fairness and total throughput.

The paper serves as a good starting point for more advanced research problems in this area, e.g., considering fast fading channels and node mobility. In addition, although the current work allows for heterogeneous transmitting power, power is used as a predetermined parameter. Future work will also consider adaptive power control and the joint design of scheduling and power control. 


\section{ACKNOWLEDGEMENT}

Maggie X. Cheng is supported in part by National Science Foundation under grant ECCS-1307458. Lin Cai is supported in part by grants from the Natural Sciences and Engineering Research Council of Canada.

\section{REFERENCES}

[1] M. Cheng and Q. Ye, "Transmission scheduling based on a new conflict graph model for multicast in multihop wireless networks," in IEEE Globecom 2012, 2012.

[2] M. Cheng, Q. Ye, and L. Cai, "Cross-layer schemes for reducing delay in multihop wireless networks," Wireless Communications, IEEE Transactions on, vol. 12, no. 2, pp. 928-937, 2013.

[3] L. Cai, L. Cai, X. Shen, and J. W. Mark, "Rex: A randomized exclusive region based scheduling scheme for mmwave wpans with directional antenna," Wireless Communications, IEEE Transactions on, vol. 9, no. 1, pp. 113-121, 2010.

[4] Z. Yang, L. Cai, and W.-S. Lu, "Practical scheduling algorithms for concurrent transmissions in rate-adaptive wireless networks," in IEEE Infocom'10, Mar. 2010, pp. 1-9.

[5] M. Bilal and M. Kang, "Throughput enhancement by concurrent transmission in WPAN with multiple antennas," in 14th International Conference on Advanced Communication Technology (ICACT), Feb. 2012, pp. 67-71.

[6] Y. Sadi and S. Ergen, "Optimal power control, rate adaptation, and scheduling for UWB-based intravehicular wireless sensor networks," IEEE Trans. on Vehicular Technology, vol. 62, no. 1, pp. 219-234, Jan. 2013.

[7] L. Xu, X. Shen, and J. W. Mark, "Dynamic fair scheduling with QoS constraints in multimedia W-CDMA cellular networks," IEEE Trans. Wireless Commun., vol. 3, no. 1, pp. 60-73, Jan. 2004.

[8] Z. Liu, M. Yang, H. Dai, and J. Dai, "Concurrent transmission scheduling for multi-hop multicast in wireless mesh networks," in IEEE International conference WiCOM, 2008.

[9] M. Alicherry, R. Bhatia, and L. Li., "Joint channel assignment and routing for throughput optimization in multi-radio wireless mesh networks," in In Proc. ACM Mobicom'05, 2005, pp. 58-72.

[10] X. Li, A. Nusairat, Y. Wu, Y. Qi, J. Zhao, X. Chu, and Y. Liu., "Joint throughput optimization for wireless mesh networks," IEEE Transactions on Mobile Computing, vol. 8, no. 7, pp. 895909, 2009.

[11] J. Yuan, Z. Li, W. Yu, and B. Li., "A cross-layer optimization framework for multihop multicast in wireless mesh networks," IEEE JSAC, vol. 24, no. 11, pp. 2092-2103, 2006.

[12] D. Qian, D. Zheng, J. Zhang, N. Shroff, and C. Joo, "Distributed CSMA algorithms for link scheduling in multihop MIMO networks under SINR model," Networking, IEEE/ACM Transactions on, vol. 21, no. 3, pp. 746-759, June 2013.

[13] K. Liu, L. Cai, and X. Shen, "Exclusive-region based scheduling algorithms for UWB WPAN," IEEE Transactions on Wireless Communications, vol. 7, no. 3, pp. 933-942, 2008.

[14] B. Radunovic and J. L. Boudec, "Optimal power control, scheduling, and routing in UWB networks," IEEE Journal on Selected Areas in Communications, vol. 22, no. 7, pp. 12521270, 2004.

[15] K. Liu, L. Cai, and X. Shen, "Multi-class utility-based scheduling for UWB networks," IEEE Transactions on Vehicular Technology, vol. 57, no. 2, pp. 1176-1187, 2008.

[16] F. Kelly, A. Maulloo, and D. Tan, "Rate control for communication networks: shadow prices, proportional fairness and stability," J. Operational Research Society, vol. 49, no. 3, pp. 237-252, Mar. 1998.

[17] P. Viswanath, D. Tse, and R. Laroia, "Opportunistic beamforming using dumb antennas," IEEE Trans. Inf. Theory, vol. 48, no. 6, pp. 1277-1294, June 2002.

[18] X. Wang and L. Cai, "Proportional fair scheduling in hierarchical modulation aided wireless networks," IEEE Trans. on Wireless Communications, vol. 12, no. 4, pp. 1584-1593, April 2013.
[19] M. Hossain, M.-S. Alouini, and V. Bhargava, "Two-user opportunistic scheduling using hierarchical modulations in wireless networks with heterogenous average link gains," IEEE Trans. Commun., vol. 58, no. 3, pp. 880-889, Mar. 2010.

[20] A. Jdidi and T. Chahed, "Impact of hierarchical modulation on proportional fair in OFDMA-based networks," in ComNet, 2010.

[21] A. Agustin, J. Vidal, and O. Muoz, "Performance of downlink schedulers with superposed or orthogonal transmissions," in IEEE ICC, 2010.

[22] M. Garey and D. Johnson, Computers and Intractability, A Guide to the Theory of NP-Completeness. FREEMAN, 1974.

\section{APPENDIX}

In this section we prove that the MTS problem is NP-hard. Apparently the MTS problem $\in$ NP. The NPhardness of MTS can be established by transforming from the Maximum Weight Independent Set problem (MWIS). The cardinality version Maximum Independent Set problem (MIS) is NP-hard [22], and so is the weighted version, since the cardinality version is a subclass of the weighted version.

We first introduce a new definition.

Definition 2 (Conflict Pair): Let $R_{i}^{j}$ be the data rate of flow $i$ when being interfered by flow $j$, and $R_{j}^{i}$ be the data rate of flow $j$ when being interfered by flow $i$. If flow $i$ and flow $j$ when sharing one slot have a combined data rate $R_{i}^{j}+R_{j}^{i} \leq \max \left\{R_{i}, R_{j}\right\}$, then flow $i$ and flow $j$ are a Conflict Pair.

The reduction from an instance of MWIS to an instance of MTS is as follows: for any given graph $G=(V, E)$ with vertex weight set $W$, construct a wireless network with $2|V|$ nodes and $|V|$ flows. Each flow when not being interfered by other flows has data rate $R_{i}=W_{i}$. In addition, the inter-flow relation is configured according to the following rules:

1) If vertex $i$ and vertex $j$ are connected by an edge in the given graph, flow $i$ and flow $j$ must be a conflict pair in the wireless network.

2) Adding a new flow $k$ to a group of flows must not change the conflict pairs of existing flows. For example, if $R_{i}^{j}+R_{j}^{i} \leq \max \left\{R_{i}, R_{j}\right\}$ then $R_{i}^{j k}+R_{j}^{i k} \leq \max \left\{R_{i}^{k}+R_{j}^{k}\right\}$, and if $R_{i}^{j}+R_{j}^{i}>$ $\max \left\{R_{i}, R_{j}\right\}$ then $R_{i}^{j k}+R_{j}^{i k}>\max \left\{R_{i}^{k}+R_{j}^{k}\right\}$.

Since channel condition, transmitting power, antenna characteristics, and node location can be arbitrarily set, we can ensure that the constructed wireless network comply with the above two rules. Thus each instance of the MWIS problem is transformed to an instance of the MTS problem in polynomial time. The optimal solution to the MTS problem is a subset of flows that has the maximum combined data rate, corresponding to the subset of independent vertices in the graph that has the maximum weight.

This completes the proof that MTS is NP-hard. 